\title{
Intermodal Bragg-Scattering Four Wave Mixing in Silicon Waveguides
}

\author{
Cosimo Lacava, Mohamed A. Ettabib, Thalia Dominguez Bucio, Graham Sharp, Ali Z. Khokhar, Yongmin Jung, \\ Marc Sorel, Frederic Gardes, David J. Richardson, Periklis Petropoulos, Francesca Parmigiani
}

\begin{abstract}
We demonstrate optical wavelength conversion in a multi-mode silicon waveguide using four wave mixing (FWM) Bragg scattering (BS) enabled by a dual-pump CW scheme. The original signal and the generated idler pair excite one spatial mode (first TE mode), while the two pumps excite a different spatial mode (second TE mode) of the same waveguide. Our approach exploits the differences in the group velocities of the various supported spatial modes to ensure phase matching only for the desired nonlinear process. In this proof-of-principle experiment, any unintended idlers are generated with an extinction ratio up to $12 \mathrm{~dB}$ relative to the phase-matched idlers for a pumps-to-signal-idler-pair wavelength detuning of about $70 \mathrm{~nm}$. The scalability of the scheme to achieve larger and multiple signal wavelength detunings from the pump frequencies is also discussed.
\end{abstract}

Index Terms-Nonlinear optics, wavelength conversion, intermodal four wave mixing

\section{INTRODUCTION}

Silicon photonics technology has now become mature enough to meet the requirements of future optical networks, providing scalable, high performance integrated optical components and circuits [1], [2], [3]. Nonlinear silicon photonic devices have also attracted considerable interest due to their ability to give rise to large third-order nonlinear effects at moderate pump powers, allowing for on-chip all-optical signal processing functionalities, such as wavelength conversion and routing, all-optical switching and format conversion [4], [5], [6]. All-optical wavelength conversion in a $\chi(3)$ nonlinear medium is typically performed exploiting four wave mixing (FWM) processes. Among the various types of FWM schemes, Bragg scattering (BS) enables the generation of either redshifted or blue-shifted copies of the original signal, $I_{B S, r}$ or $I_{B S, b}$, respectively, ideally in a noiseless manner [7], [8]. The frequencies of $I_{B S, r}$ or $I_{B S, b}$, respectively, are determined by the energy conservation law, i.e. they appear at $\omega_{s} \pm \Delta \omega$, where $\omega_{s}$ is the signal frequency and $\Delta \omega$ is the pumpto-pump wavelength detuning as illustrated in Fig.1. The arrows indicate gain (up) and loss (down) of the photon energy. As in any FWM-based process, efficient conversion is only guaranteed when phase matching among the interacting

C. Lacava, M.A. Ettabib, T. Dominguez Bucio, F. Gardes, A.Z Khokhar, Y. Jung, D.J. Richardson, P. Petropoulos are with the Optoelectonics Research Centre, University of Southampton, Highfield Avenue, SO17 1BJ, Southampton (UK)

G. Sharp and M. Sorel are with the School of Engineering, University of Glasgow, Oakfield Avenue, G12 8LP, Glasgow (UK)

F. Parmigiani was with the Optoelectonics Research Centre, University of Southampton, Highfield Avenue, SO17 1BJ, Southampton (UK). She is now with Microsoft Research UK, CB1 2FB Cambridge (UK).

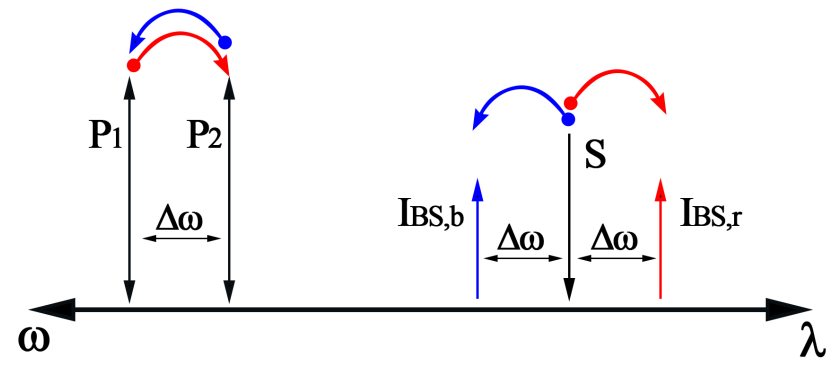

Fig. 1. Illustration of the BS-FWM process. Arrows pointing UP indicate gain of energy, arrows pointing DOWN indicate loss of energy.

waves is satisfied. This is usually achieved by tailoring the waveguide geometry to engineer the group velocity dispersion [9]. In single mode waveguides, efficient BS FWM typically requires the zero dispersion frequency to be in the middle of the spacing between the pairs of pump, and the signal and idler. When integrated devices are used, due to their shorter length scale compared to fiber-based systems, the low dispersion requirements to achieve phase matching are relaxed with many demonstrations reported in literature [9], [4], [10], [5]. Unfortunately, this implies that a variety of nonlinear processes will occur simultaneously and they typically result in undesired interference if wavelength division multiplexing telecommunication signals are to be processed. In [11] this issue was overcome by demonstrating the uni-directionality of the FWM BS process by exploiting the two polarization modes of a single mode birefringent silicon waveguide. In this work, we demonstrate the uni-directionality of the FWM BS process by exploiting the spatial modes of a multi-mode silicon waveguide. The extra degree of freedom given by the capability to carefully excite and control higher order modes in multi-mode nonlinear waveguides offers more options to fulfill the required phase matching [12], [13], [14], [15], [16]. Thus, multiple discrete spectral bands can be simultaneously phase matched, leading to simple scalable and controllable discrete wavelength converters that can be largely detuned from the pump(s) wavelengths by exciting phase matched and dispersion tailored modes of a single multi-mode nonlinear waveguide. One first preliminary demonstration of inter-modal FWM in a multimode silicon waveguide was reported in [17], where spontaneous and stimulated degenerate FWM between different modes was observed using a high peak power pspulse pump source. Herein, we demonstrate, for the first time, inter-modal FWM BS in a dispersion engineered multi-mode silicon waveguide using $\mathrm{CW}$ pumps. The original signal and 


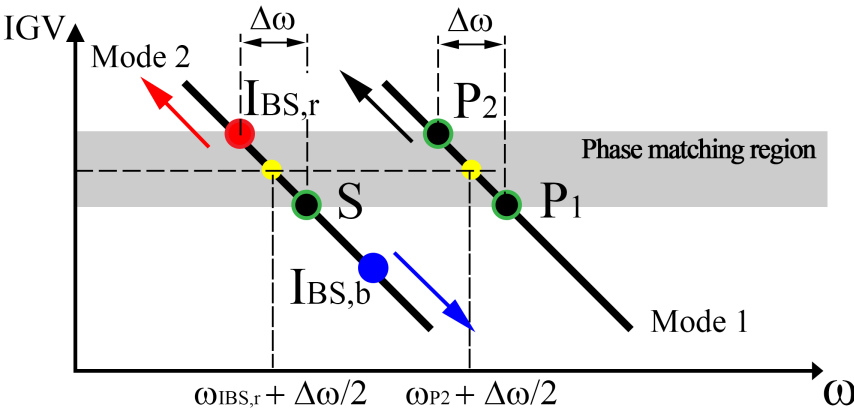

Fig. 2. Phase matching schematic for a two mode-based BS FWM device. By moving $P_{2}$ to smaller frequency values (longer wavelength) phase matching is retained for $I_{B S, r}$ only.

the newly generated idler excite one spatial mode, while the two pumps excite a different spatial mode of the same waveguide. Phase-matched signal frequency shifts of up to $18 \mathrm{~nm}$ are demonstrated for a pump-to-pump spacing of 1 $\mathrm{nm}$ with extinction ratios up to $12 \mathrm{~dB}$ between the intended and un-intended directions. In this demonstration the signal-topump detuning was of the order of $70 \mathrm{~nm}$ exciting the TE00TE10 modes, with our simulations predicting a detuning of up to $500 \mathrm{~nm}$ by exciting the TE00-TE30 modes of the same multi-mode waveguide.

\section{PRINCIPLE OF OPERATION AND WAVEGUIDE MODELING}

Referring to Fig. 1 in our experiment, the two pumps, $P_{1}$ and $P_{2}$, were launched into the fundamental TE00 mode, while the signal and the generated idlers were in the first order, TE10, mode. A cartoon of the operational principle of how to achieve phase matched inter-modal FWM for the $I_{B S, r}$ or $I_{B S, b}$, respectively, is illustrated in Fig.2 [13], [12], [18], [19], where the inverse group velocity $\left(v_{g}^{-1}\right)$ curves of the modes supported in a multi-mode waveguide are shown as a function of wavelength. Phase matching is satisfied when the following equation is (almost) fulfilled:

$$
-\beta^{0}\left(\omega_{P 1}\right)+\beta^{1}\left(\omega_{S}\right)+\beta^{0}\left(\omega_{P 2}\right)-\beta^{1}\left(\omega_{B S, r}\right)=0
$$

where $\beta^{0}(\omega)$ and $\beta^{1}(\omega)$ indicate the propagation constants of the two spatial modes involved in the nonlinear process, 0 and 1 , at the frequency $\omega$, respectively, and $\omega_{i}$ is the frequency of pump 1, pump 2, signal and red-shift BS idler for $i=\mathrm{P} 1, \mathrm{P} 2, \mathrm{~S}$ and $\mathrm{BSr}$, respectively. Eq. 1 can be conveniently rewritten as:

$$
\begin{gathered}
\beta^{0}\left(\omega_{P 1}\right)-\beta^{0}\left(\omega_{P 2}\right)=\beta^{1}\left(\omega_{S}\right)-\beta^{1}\left(\omega_{B S, r}\right) \\
\beta^{0}\left(\omega_{P 1}\right)-\beta^{0}\left(\omega_{P 1}-\Delta \omega\right)=\beta^{1}\left(\omega_{S}\right)-\beta^{1}\left(\omega_{S}-\Delta \omega\right)
\end{gathered}
$$

For small frequency detuning $(\Delta \omega \approx 0)$ Eq. 3 indicates that phase matching is achieved when the derivative function of the propagation constant, which is the inverse group velocity (IGV), in one mode, calculated at $\omega_{P 2}$, is the same as that in the other mode, calculated at $\omega_{S}$ [19]. In other words, any horizontal line crossing the group velocity curves of the various modes illustrates the phase matching across the various modes, see Fig. 2, giving an idea of the corresponding wavelengths to use. As $P 2$ is tuned to smaller frequencies ( $\Delta \omega$ increases), the $I_{B S, r}$ frequency moves in the same direction

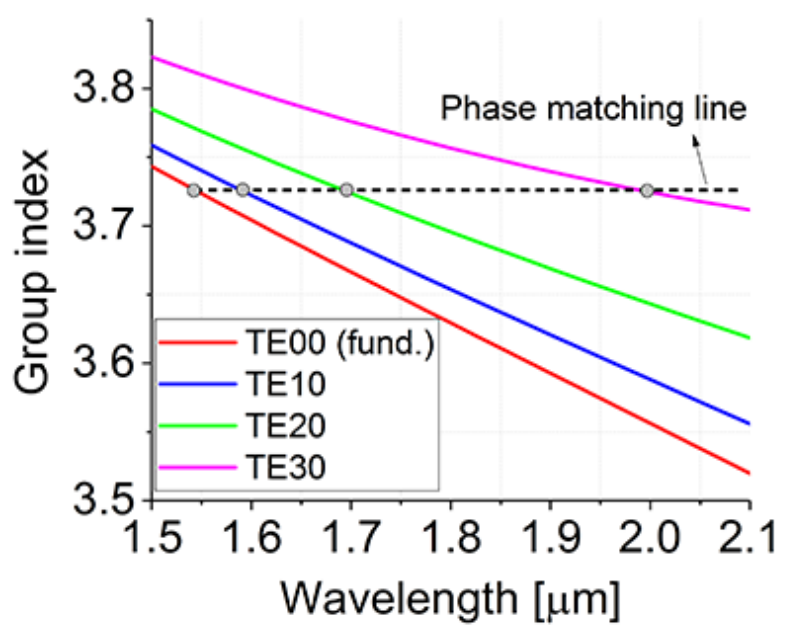

Fig. 3. Group velocity curves for the different modes propagating in a multimode silicon waveguide.

and an up-shifted horizontal line can still be drawn to cross the two $v_{g}^{-1}$ points evaluated at the new average wavelengths, if each $v_{g}^{-1}$ curve is an exact replica of the other, see Fig. 2. This implies that this process is phase matched for as long as the two $v_{g}^{-1}$ curves are an exact replica of each other. On the other hand, the frequency of $I_{B S, b}$, which also satisfies the energy conservation law, shifts to greater frequencies as $\omega_{P 2}$ decreases and only an oblique line can be drawn to cross the two $v_{g}^{-1}$ points at the new corresponding average wavelengths, implying that this process quickly moves away from phase matching. It is worth noting that if it is the frequency of $P 1$ that is tuned, the opposite discussion applies and it is the $I_{B S, b}$ of the process that is broadband, while $I_{B S, b}$ quickly phase mismatches as the detuning increases.

We initially carried out the modelling of a 1-cm long silicon on insulator (SOI) multi-mode waveguide with a cross section of $5 \times 0.22 \mu \mathrm{m}$ using the FIMMWAVE suite, and the modal properties of the waveguide were calculated using a finite difference method (FDM) solver. The numerical results of the relative group index curves $\left(n_{g}=v_{g}^{-1} c\right)$ for the first four modes are reported in Fig. 3, with the simulation predicting up to 10 supported modes in the TE-polarization. The figure shows the possibility to achieve phase matching across multiple discrete wavelength bands, from $1.55 \mu \mathrm{m}$ up to $2 \mu \mathrm{m}$, by exciting the appropriate high order modes.

The efficiency of the inter-modal FWM processes depends (quadratically) on the spatial overlap of the interacting modes $Q_{p l m n}$, where $p, l, m$ and $n$ are the modes that the interacting waves are exciting. If the two first order modes are considered (TE00 and TE10) $Q$ is non zero for the following cases only [20]:

- 4 waves in the TE00 mode

- 4 waves in the TE10 mode

- 2 waves in the TE00 mode and 2 waves in the TE10 mode

In our configuration, 2 waves (pumps) were placed in TE00 mode and two waves (signal and idler) in TE10 mode. In this 


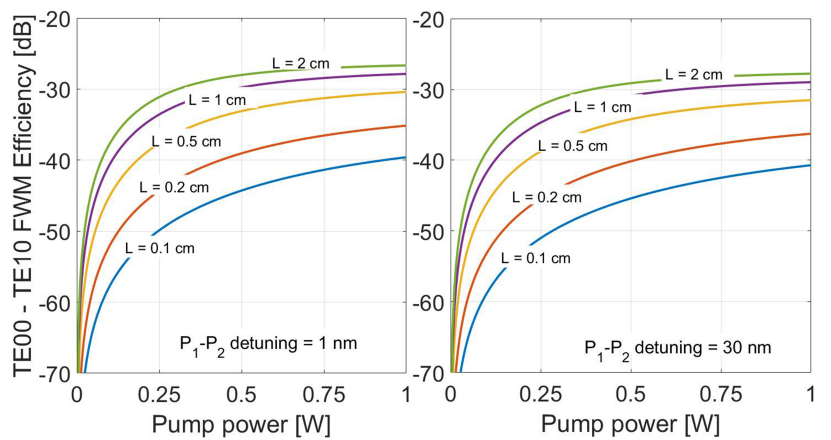

Fig. 4. Intermodal FWM numerical results for two different pump to pump detuning values: $1 \mathrm{~nm}$ (left panel) and $30 \mathrm{~nm}$ (right panel).

case $Q_{0101}$ can be calculated as:

$$
Q_{0101}=\frac{\int\left|E_{0}\right|^{2}\left|E_{1}\right|^{2} d x d y}{\int\left|E_{0}\right|^{2} d x d y \int\left|E_{1}\right|^{2} d x d y}
$$

where $E_{0}$ and $E_{1}$ represent the two mode fields of the corresponding waves (waves in TE00 and TE10, respectively). The calculated $Q_{0101}$ for our waveguide was $0.67 \mu \mathrm{m}^{-2}$. On the other hand, the calculated overlap integral for intra-modal FWM processes (four waves in the TE00 mode) was $Q_{0000}$ $=1.03 \mu \mathrm{m}^{-2}$, thus the inter-modal process leads to a $33 \%$ reduction in $Q$. We estimate that this translates into a $3.5 \mathrm{~dB}$ penalty in conversion efficiency with respect to the intra-modal process (when both process are perfectly phase-matched). By considering the waveguide geometry reported above, we modeled the intermodal FWM process in the silicon waveguide using the split step Fourier method [21]. The intermodal FWM process (as depicted in Fig. 1) can be described by the following coupled equations:

$$
\begin{aligned}
\frac{d A_{1}}{d z}= & -\frac{1}{2}\left(\alpha_{L I N}+F_{1}\right) A_{1} \\
& +i\left[\gamma_{P}\left|A_{1}\right|^{2}\right. \\
& \left.+2\left(\gamma_{P}\left|A_{2}\right|^{2}+\gamma_{M}\left|A_{3}\right|^{2}+\gamma_{M}\left|A_{4}\right|^{2}\right)\right] A_{1} \\
& +2 i \gamma_{M} A_{4} A_{3} A_{2}^{*} e^{i \Delta k z} \\
\frac{d A_{2}}{d z}= & -\frac{1}{2}\left(\alpha_{L I N}+F_{2}\right) A_{2} \\
& +i\left[\gamma_{P}\left|A_{2}\right|^{2}\right. \\
& \left.+2\left(\gamma_{P}\left|A_{1}\right|^{2}+\gamma_{M}\left|A_{3}\right|^{2}+\gamma_{M}\left|A_{4}\right|^{2}\right)\right] A_{2} \\
& +2 i \gamma_{M} A_{4} A_{3} A_{1}^{*} e^{i \Delta k z} \\
\frac{d A_{3}}{d z}= & -\frac{1}{2}\left(\alpha_{L I N}+F_{3}\right) A_{3} \\
& +i\left[\gamma_{S}\left|A_{3}\right|^{2}\right. \\
& \left.+2\left(\gamma_{M}\left|A_{1}\right|^{2}+\gamma_{M}\left|A_{2}\right|^{2}+\gamma_{S}\left|A_{4}\right|^{2}\right)\right] A_{3} \\
& +2 i \gamma_{M} A_{1} A_{2} A_{4}^{*} e^{i \Delta k z} \\
\frac{d A_{4}}{d z}= & -\frac{1}{2}\left(\alpha_{L I N}+F_{4}\right) A_{4} \\
& +i\left[\gamma_{S}\left|A_{4}\right|^{2}\right. \\
& \left.+2\left(\gamma_{M}\left|A_{1}\right|^{2}+\gamma_{M}\left|A_{2}\right|^{2}+\gamma_{S}\left|A_{3}\right|^{2}\right)\right] A_{4} \\
& +2 i \gamma_{M} A_{1} A_{2} A_{3}^{*} e^{i \Delta k z}
\end{aligned}
$$

(6)
4 also reveals that the phase matching condition is retained, even when the pump-to-pump detuning value is $30 \mathrm{~nm}$ (see Fig. 4 - right panel). It is well known that when relatively highpower is used $(P>0.5 \mathrm{~W})$ to stimulate nonlinear effects in silicon devices, free carriers generated through TPA strongly

TABLE I

PHYSICAL VALUES USING IN NUMERICAL SIMULATIONS

\begin{tabular}{ll}
\hline$Q_{0000}$ & $1.03{\mu m^{-2}}^{-2}$ \\
$Q_{1111}$ & $1.025 \mathrm{\mu m}^{-2}$ \\
$Q_{0101}$ & $0.67 \mu \mathrm{m}^{-2}$ \\
$\alpha_{L I N}$ & $1.5 \mathrm{~dB} / \mathrm{cm}$ \\
$n_{2}$ & $1.2 \times 10^{-18} \mathrm{~m}^{2} \mathrm{~W}^{-1}$ \\
$\beta_{T P A}$ & $0.68 \mathrm{~cm} / \mathrm{GW}$ \\
\hline
\end{tabular}




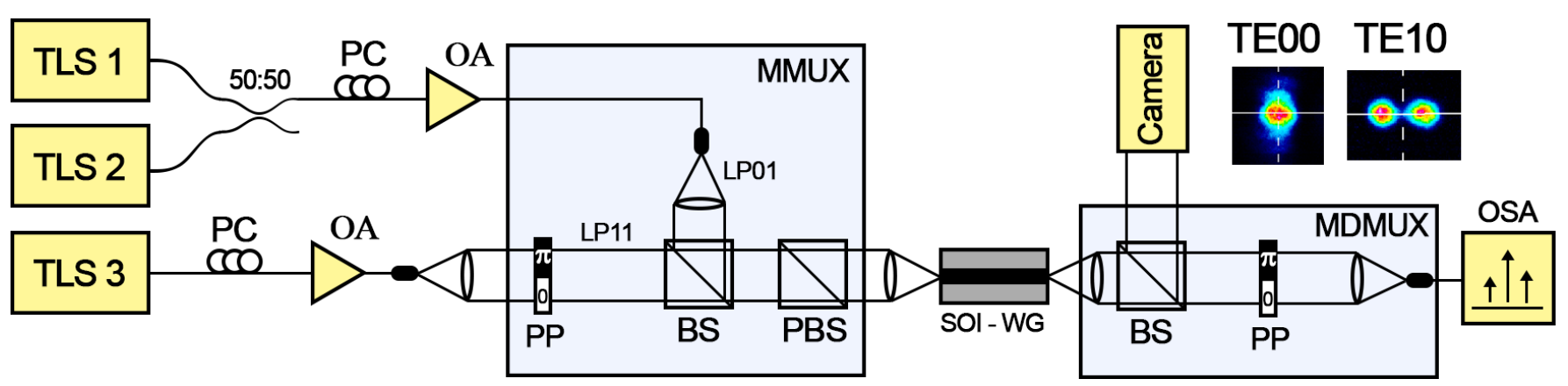

Fig. 6. BS FWM experimental set-up. Inset figures: spatial modes distributions at various points in the system.

limit the achievable FWM as they induce additional losses, further reducing the nonlinear effective length [21], [24]. As shown in [24], this detrimental effect can be mitigated by shortening the effective carrier lifetime [25], [24]. Although our fabrication capabilities did not allow for the realization of p-i-n junction waveguides, we studied the effect that a reduced carrier lifetime would have on the intermodal FWM efficiency. In Fig. 5 we report results for a $L=1 \mathrm{~cm}$ - long waveguide, for three different effective carrier lifetime values $(5,0.5$ and $0.05 \mathrm{~ns})$. The simulations show that, when high power is injected $(\approx 1 \mathrm{~W})$ the FWM efficiency can be increased by $7 \mathrm{~dB}$ if $\tau$ is shortened of a factor of 100 . Given the simulation results reported in Fig. 4, we selected $L=1 \mathrm{~cm}$ as waveguide length, thus providing a good compromise between device compactness and FWM efficiency (see Fig.4).

\section{EXPERIMENTAL SET-UP AND RESULTS}

The corresponding SOI multi-mode waveguide $(L=1 \mathrm{~cm})$ was fabricated using e-beam lithography followed by a single dry etching step and was cleaved to provide good quality waveguide-access facets. The propagation losses for both the TE00 and TE10 modes, which are the only modes used in this experiment, were measured to be $1.5 \mathrm{~dB} / \mathrm{cm}$ and the coupling losses were assessed to be of about $7.6 \mathrm{~dB} /$ facet and $8.1 \mathrm{~dB} /$ facet for the TE00 and TE10 modes, respectively. The experimental set-up used to perform the integrated intermodal FWM wavelength converter characterization is shown in Fig. 6. Two C-band CW pump signals were coupled at the input of an optical amplifier (OA) before being free-space coupled via a beam splitter (BS) with a tunable L-band signal. This allowed us to maintain the pumps in the fundamental mode (LP01), while the signal was converted into the LP11 mode using a mode-multiplexer (MMUX) based on a bulk optic phase plate (PP), which shifted the phase of half of the beam front in the transverse plane by $\pi$. All the waves were linearly polarized relative to each other and aligned with the TE polarization of the waveguide using polarization controllers and a polarization beam-splitter (PBS). The optical beam was coupled to the waveguide by using a 40x objective, by means of the end-fire coupling technique. The LP01 mode excited the fundamental TE waveguide mode (TE00), while the LP11 mode excited the TE10 waveguide mode, see corresponding inset images of Fig. 6 for their spatial mode distributions after propagation in the waveguide. The beams at the output of the waveguide were coupled back to a single-mode fiber using a second 40x objective and a mode de-multiplexer. In order to de-multiplex the two spatial modes (TE00 and TE10), a second bulk optic PP was placed before the output SM-fiber. The demultiplexer allowed extinction of either the TE00 or the TE10 optical mode, with a measured modal purity of, at least, $10 \mathrm{~dB}$. Output optical spectra were recorded using an optical spectrum analyzer (OSA). Note that to measure intra-modal FWM, the PPs were removed both at the input and output of the set-up to guarantee that all the signal waves were exciting only the fundamental mode of the waveguide which was then properly coupled into the output SM fiber.

According to our numerical simulations (please also refer to Fig. 3), phase matching can occur between waves exciting the TE00 and TE10 modes when their wavelength separation is $\approx 65 \mathrm{~nm}$. To prove this, we initially placed the two pumps at wavelengths of $\lambda_{P 1}=1544.75 \mathrm{~nm}$ and $\lambda_{P 2}=1545.25 \mathrm{~nm}$, respectively, while the signal was tuned from $1580 \mathrm{~nm}$ to 1620 $\mathrm{nm}$, limited by our available tunable source. Fig. 7 shows the conversion efficiency as a function of the signal wavelength of the red-shifted idler, for both the inter- and intra-modal FWM processes (blue and red dots, respectively) when a pump power of $70 \mathrm{~mW}$ was used. As shown in the figure, inter-modal FWM resulted in up to $12 \mathrm{~dB}$ higher conversion efficiency relative to intra-modal FWM, despite the larger nonlinear cofficient

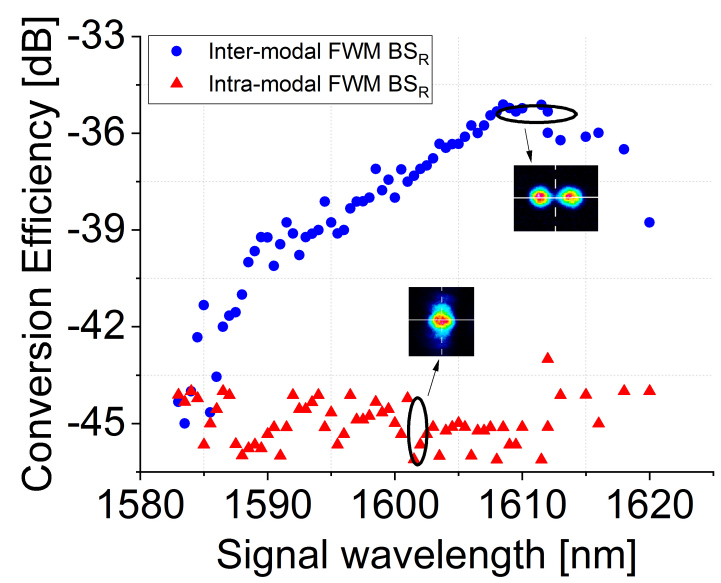

Fig. 7. Conversion efficiency as a function of the signal wavelength for intramodal FWM (red-curve) and inter-modal FWM (blue curve) processes. 


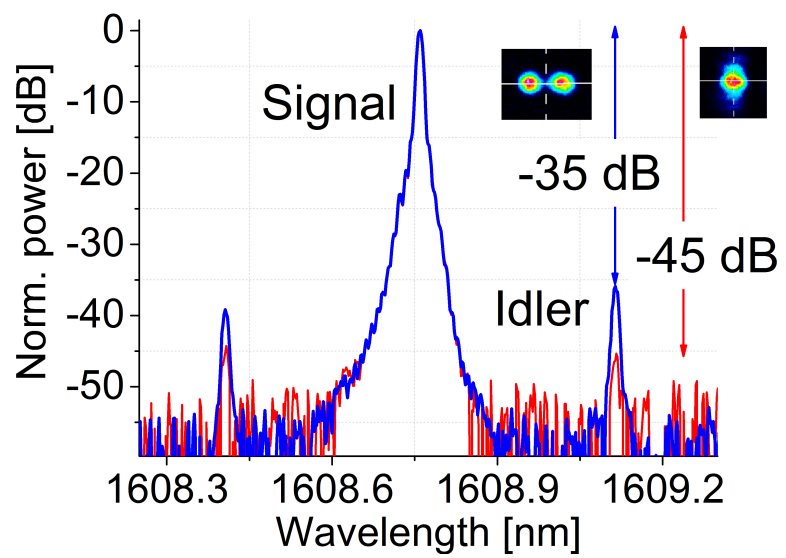

Fig. 8. Examples of typical spectra at the output sample after inter(blue curve) and intra- (red curve) modal FWM processes when the signal wavelength is set to $1608.67 \mathrm{~nm}$.

of the intra-modal case. This is because phase matching was only satisfied for the inter-modal case at the optimal signal wavelength of $\lambda_{s}=1608.7 \mathrm{~nm}$, i.e. for a pumps-tosignal detuning of about $65 \mathrm{~nm}$. As the signal wavelength was detuned from its optimum, the conversion efficiency dropped achieving a measured $-3 \mathrm{~dB}$ half-bandwidth of about $9 \mathrm{~nm}$. Assuming the whole bandwidth to be symmetric an overall $-3 \mathrm{~dB}$ bandwidth of $18 \mathrm{~nm}$ is estimated. The intra-modal FWM results (Fig. 7, red triangles) show relatively constant values as a function of signal wavelength due to the short length of the sample (i.e. showing low net dispersion), with a small increase of efficiency as the signal was tuned closer to the pump wavelengths as the process becomes better phase matched.

Fig. 8 shows the signal and the generated idler spectra when a pump power of $120 \mathrm{~mW}$ was coupled into the waveguide both for the intra- and inter-modal FWM processes. The measured inter-modal FWM efficiency (phase-matched) was $-35 \mathrm{~dB}$ for $I_{B S, r}$ and $-37 \mathrm{~dB}$ for $I_{B S, b}$ (blue line in Fig. 8). The intra-modal generated idlers (showing no phase-matching) were also measured (red line in Fig. 8), revealing a conversion efficiency of about $-45 \mathrm{~dB}$ in both the $I_{B S, r}$ and the $I_{B S, b}$ frequencies.

The inter-modal phase matching bandwidth was also assessed by measuring the FWM-efficiency as a function of $\Delta \omega$ for both the red- and blue-shifted idlers as shown in Fig. 9. In this case we kept $\lambda_{P 1}$ and $\lambda_{S}$ constant to 1544.75 $\mathrm{nm}$ and $1608.6 \mathrm{~nm}$, respectively, while $\lambda_{P 2}$ was tuned. As previously discussed the operational bandwidth is not the same for the two processes, with the red-shifted idler generation showing a broader operation bandwidth: its $-3 \mathrm{~dB}$ bandwidth is $7 \mathrm{~nm}$ against $4 \mathrm{~nm}$ for the blue-shifted one. This can be very interesting for controlled wavelength conversion, when only one nonlinear process is desirable. In our particular case with a pump-to-pump detuning of $5.5 \mathrm{~nm}$, we obtained a redshifted idler more than $12 \mathrm{~dB}$ stronger that the blue-shifted one as shown in Fig. 9 (insets). It is also worth noting that much broader bandwidths are to be expected by properly engineering the group index of the two spatial modes such that they are shifted replicas of one another at the wavelengths of interest [14], or exhibit a mirror symmetry [26].

\section{CONCLUSION}

We demonstrated, for the first time, a dual-pump CW silicon photonic wavelength converter, using an inter-modal FWM BS process. We report a conversion efficiency of $-35 \mathrm{~dB}$, between the TE00 and the TE10 waveguide modes, when a pump power of $120 \mathrm{~mW}$ is coupled into the waveguide and a pumps-to-signal detuning of $70 \mathrm{~nm}$. When the pump-topump detuning was set to $5.5 \mathrm{~nm}$, the red-shifted idler showed more than $12 \mathrm{~dB}$ stronger conversion efficiency than the blueshifted copy. Furthermore, our simulated group index curves predict multiple phase matching bands, spanning from $1.5 \mu \mathrm{m}$ to $2 \mu \mathrm{m}$, if the signal/pumps excite different higher order modes of the same multi-mode waveguide. We note that the overall FWM BS efficiency in SOI is mainly limited by two photon absorption (TPA) and free carrier absorption (FCA) as typically happens in silicon-based integrated devices [4]. We note that in order to meet the requirements of practical applications, higher FWM efficiency levels would be required [27]. As discussed in Sec. 2, a viable option to mitigate the effect of TPA and FCA detrimental effects is to reduce the effective carrier lifetime (see Fig. 5). This could be achieved by including a p-i-n junction on the waveguide [25], allowing the device to operate at higher pump power levels with lower nonlinear losses, thus increasing the achievable FWMefficiency values. Alternative options include the use of other silicon photonics-compatible platforms instead of silicon that are less compromised by TPA and FCA effects, such as those based, for example, on silicon nitride compounds[28].
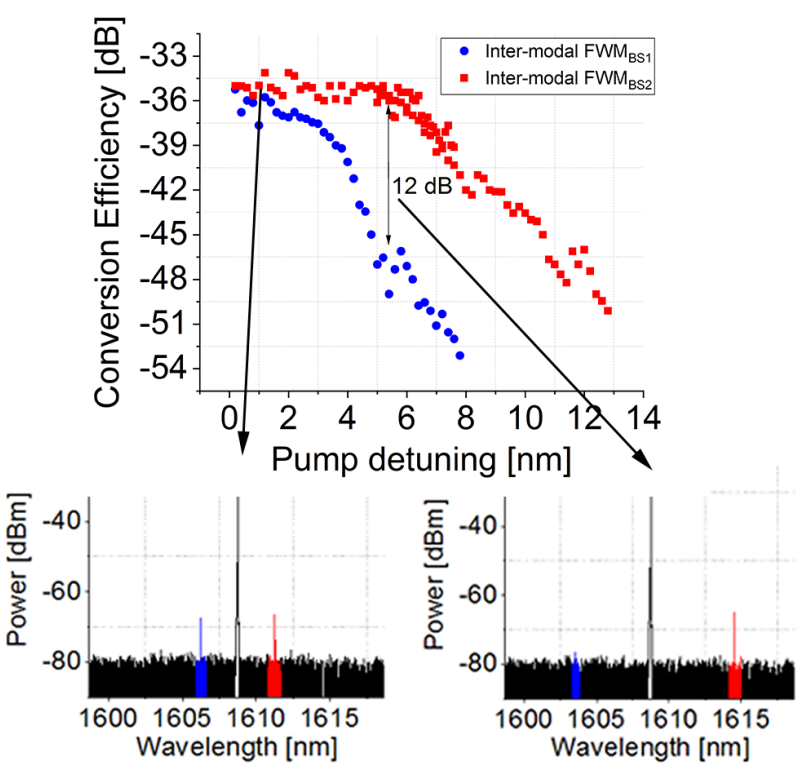

Fig. 9. Conversion efficiency as a function of the pump-to-pump detuning for inter-modal FWM, red-shifted idler (redcurve) and inter-modal FWM blueshifted idler (blue curve) processes. 


\section{ACKNOWLEDGMENT}

We would like to thank Dr. Peter Horak for useful discussions. This work was funded by The Engineering and Physical Sciences Research Council (EPSRC) through the Project Silicon Photonics for Future Systems and through the grant EP/P026575/1. http://doi.org/10.5258/SOTON/D0425

\section{REFERENCES}

[1] C. R. Doerr, "Silicon photonic integration in telecommunications," Frontiers in Physics, vol. 3, no. August, pp. 1-16, 2015.

[2] R. Marchetti, C. Lacava, A. Khokhar, X. Chen, I. Cristiani, D. J. Richardson, G. T. Reed, P. Petropoulos, and P. Minzioni, "Highefficiency grating-couplers: Demonstration of a new design strategy," Scientific Reports, vol. 7, no. 1, pp. 1-8, 2017.

[3] A. E. J. Lim, J. Song, Q. Fang, C. Li, X. Tu, N. Duan, K. K. Chen, R. P. C. Tern, and T. Y. Liow, "Review of Silicon Photonics Foundry Efforts," IEEE Journal of Selected Topics in Quantum Electronics, vol. 20, no. 4, pp. 405-416, 2014.

[4] C. Lacava, M. A. Ettabib, and P. Petropoulos, "Nonlinear Silicon Photonic Signal Processing Devices for Future Optical Networks," Applied Sciences, vol. 7, no. 1, p. 103, 2017.

[5] M. A. Ettabib, C. Lacava, Z. Liu, A. Bogris, A. Kapsalis, M. Brun, P. Labeye, S. Nicoletti, D. Syvridis, D. J. Richardson, and P. Petropoulos, "Wavelength conversion of complex modulation formats in a compact SiGe waveguide," Optics Express, vol. 25, no. 4, p. 3252, 2017.

[6] H. Hu, H. Ji, M. Galili, M. Pu, C. Peucheret, H. C. H. Mulvad, K. Yvind, J. M. Hvam, P. Jeppesen, and L. K. Oxenløwe, "Ultra-highspeed wavelength conversion in a silicon photonic chip," Optics Express, vol. 19, no. 21, p. 19886, 2011.

[7] K. Uesaka, K. K.-y. Wong, M. E. Marhic, and L. G. Kazovsky, "Wavelength Exchange in a Highly Theory and Experiments," IEEE Journal of Selected Topics in Quantum Electronics, vol. 8, no. 3, pp. 560-568, 2002.

[8] C. J. McKinstrie, J. D. Harvey, S. Radic, and M. G. Raymer, "Translation of quantum states by four-wave mixing in fibers," Optics Express, vol. 13 , no. 22, p. 9131, 2005.

[9] A. C. Turner, C. Manolatou, B. S. Schmidt, M. Lipson, M. A. Foster, Y. Okawachi, and A. L. Gaeta, "Tailored anomalous group-velocity dispersion in silicon waveguides," Optics Express, vol. 14, no. 10, pp. 4357-4362, 2006.

[10] C. Lacava, M. Ettabib, I. Cristiani, J. Fedeli, D. Richardson, and P. Petropoulos, "Ultra-Compact Amorphous Silicon Waveguide for Wavelength Conversion," IEEE Photonics Technology Letters, vol. 28, no. 4, pp. 410-414, 2016.

[11] B. Bell, C. Xiong, D. Marpaung, C. McKinstrie, and B. Eggleton, "Unidirectional wavelength conversion in silicon using four-wave mixing driven by cross-polarized pumps," Optics Letters, vol. 42, no. 9, pp. $1668-1671,2017$.

[12] F. Parmigiani, M. Guasoni, O. F. Anjum, P. Horak, Y. Jung, L. GrunerNielsen, P. Petropoulos, and D. J. Richardson, "Polarization Insensitive Wavelength Conversion in a Few Mode Fibre," in ECOC 2017, 2017, p. W2F2.

[13] F. Parmigiani, P. Horak, Y. Jung, L. Grüner-Nielsen, T. Geisler, P. Petropoulos, and D. J. Richardson, "All-optical mode and wavelength converter based on parametric processes in a three-mode fiber," Optics Express, vol. 25, no. 26, p. 33602, 2017.

[14] J. Demas, G. Prabhakar, T. He, S. Ramachandran, and S. Ramachandran, "Broadband and Wideband Parametric Gain via Intermodal Four-Wave Mixing in Optical Fiber," Conference on Lasers and Electro-Optics, no. 2, p. SM3M.1, 2017.

[15] J. Demas, L. Rishøj, X. Liu, G. Prabhakar, and S. Ramachandran, "Highpower, wavelength-tunable NIR all-fiber lasers via intermodal four-wave mixing," Optics InfoBase Conference Papers, vol. Part F43-C, pp. 100101, 2017.

[16] Y. Ding, J. Xu, H. Ou, and C. Peucheret, "Mode-selective wavelength conversion based on four-wave mixing in a multimode silicon waveguide," Optics Express, vol. 22, no. 1, p. 127, 2014.

[17] S. Signorini, M. Mancinelli, M. Bernard, M. Ghulinyan, G. Pucker, and L. Pavesi, "Broad wavelength generation and conversion with Multi Modal Four Wave Mixing in silicon waveguides," in Group IV Photonics, 2017, pp. 59-60.
[18] S. M. M. Friis, I. Begleris, Y. Jung, K. Rottwitt, P. Petropoulos, D. J. Richardson, P. Horak, and F. Parmigiani, "Inter-modal four-wave mixing study in a two-mode fiber," Optics Express, vol. 24, no. 26, p. 30338, 2016.

[19] R. Essiambre, M. A. Mestre, R. Ryf, A. H. Gnauck, R. W. Tkach, A. R. Chraplyvy, Y. Sun, X. Jiang, and R. Lingle, "Experimental Investigation of Inter-Modal Four-Wave Mixing in Few-Mode Fibers," IEEE Photon. Technol. Lett., vol. 25, no. 6, pp. 539-542, 2013.

[20] Y. Xiao, R.-J. Essiambre, M. Desgroseilliers, A. M. Tulino, R. Ryf, S. Mumtaz, and G. P. Agrawal, "Theory of intermodal four-wave mixing with random linear mode coupling in few-mode fibers," Optics Express, vol. 22, no. 26, p. 32039, 2014.

[21] G. P. Agrawal, Nonlinear Fiber Optics, 5th ed., Elsevier, Ed. New York: Academic Press, 2013.

[22] Q. Lin, O. J. Painter, and G. P. Agrawal, "Nonlinear optical phenomena in silicon waveguides: modeling and applications." Optics express, vol. 15, no. 25, pp. 16604-16644, 12 2007. [Online]. Available: http://www.ncbi.nlm.nih.gov/pubmed/19550949

[23] M. Borghi, C. Castellan, S. Signorini, A. Trenti, and L. Pavesi, "Nonlinear silicon photonics," Journal of Optics, vol. 19, p. 093002, 2017.

[24] A. Liu, R. Jones, H. Rong, A. W. Fang, M. J. Paniccia, D. Hak, and O. Cohen, "Net continuous wave optical gain in a low loss silicon-oninsulator waveguide by stimulated Raman scattering." Optics express, vol. 12, no. 18, pp. 4261-4268, 2004.

[25] H. Rong, R. Jones, A. Liu, O. Cohen, D. Hak, A. Fang, and M. Paniccia, "A continuos wave Raman silicon laser," Nature, vol. 433, no. 7027, pp. 725-728, 2005.

[26] M. Guasoni, F. Parmigiani, and D. J. Richardson, "Novel fiber design for wideband conversion and amplification in multimode fibers," in ECOC 2017, 2017.

[27] T. Inoue, H. Nguyen Tan, K. Tanizawa, S. Petit, K. Ota, S. Takasaka, T. Yagi, and S. Namiki, "Recent Progress and Challenges in Developing Practical All-optical Wavelength Converter," in Asia Communications and Photonics Conference 2015, 2015, p. AS3J.2.

[28] C. Lacava, S. Stankovic, A. Khokhar, T. Bucio, F. Gardes, G. Reed, D. Richardson, and P. Petropoulos, "Si-rich Silicon Nitride for Nonlinear Signal Processing Applications," Scientific Reports, vol. 7, no. 1, 2017. 